\title{
THE GALACTIC GLOBULAR CLUSTER SYSTEM
}

\author{
JAMES E. HESSER \\ Dominion Astrophysical Observatory \\ Herzberg Institute of Astrophysics \\ National Research Council of Canada \\ Victoria, B.C. V8X 4M6 Canada \\ (hesser@dao.nrc.ca)
}

\section{Introduction}

"Although the evidence presented in the preceding discussion is still very fragmentary, there can be no doubt that, in dealing with galaxies, we have to distinguish two types of stellar populations, one which is represented by the ordinary H-R diagram (type I), the other by the H-R diagram of the globular clusters (type II). Characteristic of the first type are highly luminous $O$ - and $B$-type stars and open clusters, of the second, globular clusters and shortperiod Cepheids...it should be pointed out that these same two types of stars were recognized by Oort as early as 1926. Oort showed that the high-velocity stars of our galaxy (our type II) are of a kind quite different from the slowmoving stars (type I) which predominate in the solar neighborhood."

Walter Baade's (1944) epochal words continue to profoundly affect astronomy, as will be abundantly clear throughout this Symposium, for which my introduction will address three questions:

1. How representative of Pop II are the Milky Way's globular clusters (GCs)?

2. How stable are the GC system's chemical and dynamical properties...or, better, our knowledge thereof?

3. How did the system form and what does that tell us about how large spiral galaxies form?

Thus the aim is to begin with material inspired by Baade's definition of Pop II, and to finish with new results from the Hubble Space Telescope (HST) and from modern ground-based facilities that promise answers, perhaps definitive at last, to the third query. 


\section{Question One: How Representative of Pop II Are the Milky Way's GCs?}

It is worth recalling that the luminous halo of the Galaxy (and its intimate association with the Pop II concept) contains not only the prominent Messier GCs evoked upon reading Baade's words, but also the virtually invisible, "fluffy" dwarf spheroidal (dSph) galaxies as well as a population of field stars that outnumbers the luminous mass in GCs by about 100 .

Since the early 1980s color-magnitude diagrams (CMDs) have improved spectacularly. For the mostly metal-deficient Messier clusters whose upper giant-branch properties so influenced Baade, precision photometry now extends from the giant branch tip to five magnitudes down the hydrogenburning main sequence (e.g., M15: Durrell and Harris, 1993). The same technologies, combined with powerful Southern hemisphere telescopes, provide for the first time CMDs for the metal-rich clusters superposed upon the dense star fields of the Galactic bulge. For some we find (e.g., Ortolani et al., 1993) that the coolest giants are so heavily blanketed in V-light that their locus doubles over to reach again the level of the horizontal branch (HB).

Rather little was known about dSphs from the properties of the two recognized in the early $1940 \mathrm{~s}$, but interesting differences emerge from comparisions today of their global properties with those of the GCs, Table 1. (This table stems from many literature references, notably Pryor (1992), and is meant to be illustrative, rather than definitive: e.g., exceptional GCs like $\omega$ Cen are ignored.)

We are fairly confident that our inventory of GCs is nearly complete (e.g., Racine and Harris, 1989), but the discovery of the disrupting Sagittarius dSph (Ibata et al., 1994) suggests the same may not be true for $\mathrm{dSphs}$. While the outer halo GCs populate the same volume of space as the innermost dSphs, some properties of these objects differ dramatically, e.g., their core radii $\left(\mathrm{r}_{\mathrm{c}}\right)$. The overlap in $\mathrm{M}_{\mathrm{V}}$ is partial, as is that in heavyelement composition, herein denoted by the usual logarithmic difference with respect to the Sun, $[\mathrm{Fe} / \mathrm{H}]$. Within a given $\mathrm{GC}$, the internal composition range is, essentially, below detection thresholds for elements other than $\mathrm{CNO}, \mathrm{Na}$ and $\mathrm{Al}$, but $\mathrm{dSphs}$ exhibit quite measurable ranges. Moreover, in $\mathrm{dSphs}$ the mean metallicity increases with increasing $\mathrm{MV}_{\mathrm{V}}$. Together these composition differences suggest that $\mathrm{dSphs}$ can be self-enriched, while most GCs are unlikely to be. The large $M / L_{V}$ ratios for $d S p h s$ indicate that they contain substantial amounts of dark matter (DM); the M/L ratios decrease with increasing L, while no such behavior is found for the GCs. Finally, the presence of anomalous Cepheids and carbon stars suggests that most dSphs possess a stellar population component younger than that of the GCs. (The issue of systemic age ranges will be the focus of $\S 4$.) 
TABLE 1. Some Comparisons of Globular Clusters and Dwarf Spherodials

\begin{tabular}{lll}
\hline Property & Globular Clusters & Dwarf Spherodials \\
\hline Number & $\sim 150$ & $\geq 9$ \\
$\mathrm{R}_{\mathrm{gc}}(\mathrm{kpc})$ & $0.9 \leftrightarrow 120$ & $70 \leftrightarrow 270$ \\
$\mathrm{r}_{\mathrm{c}}(\mathrm{pc})$ & $0.1 \leftrightarrow 27$ & $150 \leftrightarrow 530$ \\
$\mathrm{M}_{\mathrm{V}}$ & $-1.7 \leftrightarrow-10.4$ & $-8.7 \leftrightarrow-12.4$ \\
$<[\mathrm{Fe} / \mathrm{H}]>$ & $-2.2 \leftrightarrow \sim 0$ & $-2.1 \leftrightarrow-1.4$ \\
{$[\mathrm{Fe} / \mathrm{H}]$ range $(\mathrm{dex})$} & $\leq 0.03$ & $\sim 0.25$ \\
$\mathrm{M} / \mathrm{L} \mathrm{V}$ & $\sim 0.8 \leftrightarrow 3.0$ & $\sim 5.7 \leftrightarrow 94$ \\
Internal DM? & No & Yes \\
Anomalous Cepheids? & No & Yes \\
Carbon Stars? & No & Yes \\
Internal Age range? & No & Yes \\
Systemic Age range? & Yes & Yes \\
\hline
\end{tabular}

Our understanding of the field halo star population has also dramatically advanced since Baade. Various studies (e.g., Harris, 1987; Saha, 1985) have demonstrated that the spatial distributions of the GCs and of the field RR Lyrae stars have similar form (space density $\propto R_{\mathrm{gc}}{ }^{-3.5}$ for $\mathrm{R}_{\mathrm{gc}} \leq 30 \mathrm{kpc}$ ). Similarly, the metallicity distribution functions are comparable over the entire $R_{\mathrm{gc}}$ range, but for $\mathrm{R}<\mathrm{R}_{\mathrm{o}}$ the field $\mathrm{R} R$ Lyrae stars are more metal rich than the cluster ones (Suntzeff et al., 1991). The latter authors also find evidence for a $-0.06 \mathrm{dex} \mathrm{kpc}^{-1}$ gradient in $[\mathrm{Fe} / \mathrm{H}]$ interior to the solar circle, and none exterior to it. They conclude the GCs account for $\sim 2 \%$ of the luminous mass of the Galactic halo, which they estimate to be $\sim 9 \times 10^{8} \mathrm{M}_{\odot}\left(4<\mathrm{R}_{\mathrm{gc}}<25 \mathrm{kpc}\right)$. Djorgovski and Meylan (1994) note that $\mathrm{R}_{\mathrm{gc}}{ }^{-3.5}$ is much steeper than the $\sim \mathrm{R}^{-2}$ density law of a dark matter halo that yields a flat rotation curve. What evolutionary process, they ask, converts the GC law from shallow to steeper over a Hubble time without doing the same for DM?

Moreover, systematic surveys of metallicities have shown ( $99 \%$ confidence) that the metallicity distribution function of field halo dwarf stars (specifically those on retrograde orbits) extends to lower $[\mathrm{Fe} / \mathrm{H}]$ than does that of the GCs (Laird et al., 1993).

We asked: How representative of Pop II are the Milky Way's globular clusters? The foregoing - and much of what follows - indicates how much more varied and complex the GCs are than was perhaps apparent in Baade's time, and how much has been learned since then (see also Stetson, 1993), e.g.: 
- Not all old stars are metal-poor, nor are all young ones metal-rich;

- Not all clusters of globular appearance are old ( $c f$. the LMC, SMC, M33....);

- GC CMDs exhibit a complicated dependence on $[\mathrm{Fe} / \mathrm{H}]$ and other, poorly understood parameters;

- The Galactic halo contains some 100 times more luminous matter in field stars than in GCs;

- dSphs have multiple generations of stars, while GCs at similar $\mathbf{R}_{\mathrm{gc}}$ do not; and

- Metallicity distribution functions of halo stars extend to lower $[\mathrm{Fe} / \mathrm{H}]$ than do either those of the GCs or dSphs.

Even though the CMDs of Galactic GCs initially contributed to the definition of Population II, it is unlikely that those CMDs or clusters represent all relevant characteristics of the concept this Symposium addresses.

3. Question Two: How Stable Are the GC System's Chemical and Dynamical Properties...or, Better, our Knowledge Thereof?

Abundances are tightly linked to determinations of ages in the Galactic halo (§4), as they are to discovering (from element and isotope ratios) the mix of type I and II supernovae ejecta that went into the "soup" from which halo objects formed (which we won't pursue further here). How confident are we that available abundance information is adequate for reliable age determinations? And if the abundances are sound, how certain are we that the GC system (or individual clusters) haven't been so modified by dynamical effects that they no longer fairly represent the formation era of the Galaxy?

\subsection{ABUNDANCES}

Some chemical properties of the Galactic GCs seem well established, including:

$\bullet-2.2 \lesssim[\mathrm{Fe} / \mathrm{H}] \lesssim 0.0$

- The $[\mathrm{Fe} / \mathrm{H}]$ distribution is bimodal, and is reflected in the kinematics, thus implying the existence of at least two subsystems (e.g., Kinman, 1959; Zinn, 1985; Hesser et al., 1986): a) a "halo" subsystem having $[\mathrm{Fe} / \mathrm{H}] \leqslant-0.8$ and slowly rotating $\left(\sim 40 \mathrm{~km} \mathrm{~s}^{-1}\right)$, and b) a "disk" or "nuclear bulge" subsystem having $[\mathrm{Fe} / \mathrm{H}] \approx-0.8$ which is rapidly rotating $\left(\sim 190 \mathrm{~km} \mathrm{~s}^{-1}\right)$.

- A weak gradient in $[\mathrm{Fe} / \mathrm{H}]$ is found for $\mathrm{R}_{\mathrm{gc}} \leq 8 \mathrm{kpc}$, whereas none is detectable elsewhere (Searle, 1977; Searle and Zinn, 1978; Suntzeff et al., 1991; Armandroff et al., 1992).

- The absence of a correlation between cluster metallicity and luminosity in all GC systems studied (Harris, 1991), as well as the low dispersion of 
[Fe/H] within individual GCs (e.g., Suntzeff, 1993) implies that GCs were not self enriched...

- But giants in any particular GC exhibit wide ranges of CN band strengths that are often bi-modal and anti-correlated with $\mathrm{CH}$. The latter implies that products of $\mathrm{C} \rightarrow \mathrm{N}$ and/or $\mathrm{O} \rightarrow \mathrm{N}$ processing in the hydrogenburning shell (e.g., Smith, 1987; Smith and Wirth, 1991; Briley et al., 1994a) have been observed in the surface layers.

The last point is important for age determinations, since the turnoff from the main sequence is sensitive to the abundance of oxygen (VandenBerg, 1988) and/or of $O$ plus the $\alpha$ elements (Salaris et al., 1993). Highdispersion spectroscopy has yielded a number of puzzling results reviewed by Kraft (1994). For instance, among M13 giants $-0.8<[\mathrm{O} / \mathrm{Fe}]<+0.5$ ! What oxygen abundance characterizes the M13 dwarfs used in age determinations, and what physical mechanisms lead to the range of abundances and correlations thereof among evolved giants?

Much evidence favors mixing of processed material from the $\mathrm{C} \rightarrow \mathrm{N}$ and/or $\mathrm{O} \rightarrow \mathrm{N}$ burning regions of the hydrogen-burning shell to the observable surface. An example is the very orderly decrease (by $0.8 \mathrm{dex}$ ) of $[\mathrm{C} / \mathrm{Fe}]$ as one ascends the red giant branch (e.g., Briley et al., 1990). On the other hand, the similarity of the distributions of $\mathrm{CH}$ and $\mathrm{CN}$ band strengths among horizontal branch stars (Norris and Freeman, 1982) and main-sequence stars (Briley et al., 1994b; Croke, et al., this conference) for 47 Tuc strongly suggests a primordial origin. Primae facie evidence that the star-to-star differences in CNO within a given cluster must be largely of a primordial origin was provided by the observation (again, see Kraft's 1994 review) that the abundance of $\mathrm{Na}$, an element not thought to be produced during nucleosynthesis in low-mass stars, anticorrelates with O abundances. However, Langer et al.(1993), working on Denisenkov and Denisenkova's (1990) suggestion, have shown that neutron captures on ${ }^{22} \mathrm{Ne}$ in the $\mathrm{O} \rightarrow \mathrm{N}$ burning region of the H-burning shell can make ${ }^{23} \mathrm{Na}$ in some 60,000 years; thus, internal mixing during ascent of the giant branch apparently remains a viable explanation for the correlations between $\mathrm{Na}$ and CNO element ratios.

Many of us (naively!) thought that once abundances from high-dispersion spectroscopy of GC giants became available, we would pin down this parameter, which is vital to age determinations. Regretably, until we understand more fully what processes within star clusters (but not apparently in the field) lead to the range of abundances observed among luminous giants, that goal eludes us. 


\subsection{DYNAMICS}

Remarkably, GCs in the rather different environments of the Galaxy, Magellanic Clouds and Fornax dSph possess similar M/Lv, $2.5 \pm 1$ (Pryor et al., 1991; Meylan and Pryor, 1993, Dubath et al., 1992). That notwithstanding, a burgeoning area of GC astrophysics deals with how dynamical processes, including tidal capture (with induced cluster formation?) and destruction, have affected, and are affecting, the GC system.

A useful framework is depicted in Fall's (1980) "survival triangle", in which the original GC system is schematically viewed in the perspective of a triad of destruction mechanisms: disc shocking, dynamical friction and evaporation. Numerous theoretical investigations have pointed to the possible, even likely, conclusion that the present GC system is a mere shadow of the original (e.g., Aguilar et al., 1988; Chernoff and Weinberg, 1990), although van den Bergh (1994) argues that the original GC system has been reduced by only a factor $\sim 1.7$. Djorgovski and Meylan (1994) note that, even if all the original clusters started with a $\delta$-function distribution of properties, tidal shocking alone would have caused dramatic evolution of systemic properties over a Hubble time. They summarize the evidence from GC core properties that the most dynamically evolved clusters lie near the Galactic center, although there is a large scatter at all $\mathbf{R}_{\mathrm{gc}}$ (or height above the Galactic plane, $\mathrm{Z}_{\mathrm{gp}}$ ); tidal shocks at low $\mathrm{R}_{\mathrm{gc}}$ or $\mathrm{Z}_{\mathrm{gp}}$ apparently accelerate internal evolution to core collapse and evaporate low-concentration clusters.

Van den Bergh et al.(1991), guided by N-body calculations that show that the projected half light radius is approximately invariant over 10 or more relaxation times (whereas $r_{c}$ is not), demonstrated that the angular diameters of GCs increase with $R_{\mathrm{gc}}$ in the Galaxy, LMC and NGC 5128. The absence of large diameter clusters near the Galactic center could have resulted from tidal disruption, but the absence of compact clusters at $R_{\mathrm{gc}}>8 \mathrm{kpc}$ would appear to reflect the formation process.

We asked: How stable are the GC system's chemical and dynamical properties...or, better, our knowledge thereof? The answer: Not as much as perhaps is required to answer with confidence how the Galaxy formed. Dynamically, the GC system:

- Exhibits evolution in the form of correlations of GC core properties and of mass function slope with $\mathrm{R}_{\mathrm{gc}}$ (and/or, $\mathrm{Z}_{\mathrm{gp}}$ ), and

- Contains dissolving objects (e.g., E3 and the Sagittarius dSph) and/or ones whose severely truncated main-sequence luminosity functions (e.g., Pal 5) suggest significant mass loss over a Hubble time.

- Moreover, many theoretical studies suggest that the GC system today may be a mere shadow of the original. 
Chemically, the GCs exhibit:

- A subtle gradient in $[\mathrm{Fe} / \mathrm{H}]$ for $\mathrm{R} \lesssim \mathrm{R}_{\mathrm{o}}$, but none beyond, which is a clue important to the formation process;

- Evidence that $[(\mathrm{CNO}, \mathrm{Na}, \mathrm{Al}) / \mathrm{Fe}]$ in luminous giants of a cluster are not the same. The differences appear to arise from some combination of mixing during stellar evolution and primordial variations, which signifies that even high-dispersion studies of giants are not measuring abundance ratios appropriate for use in age determinations from main-sequence stars.

The solution to the latter situation lies in utilization of $8-10-\mathrm{m}$ telescopes for high-dispersion spectroscopy of main-sequence stars, and continued efforts to improve model atmosphere analyses.

\section{Question Three: How Did the System Form and What Does That Tell Us about How Large Spiral Galaxies Form?}

My focus will be on the quest for observational contraints on the relative chronology for Galactic formation, rather than on theories of collapse, cluster formation physics, or absolute ages/cosmology. (While pursuing a Galactocentric view, one ought to bear in mind that differences are apparent between cluster and star formation scenarios in other galaxies, e.g., Kumai et al., 1993.) The current debate about Galactic formation compares two simple pictures:

- The collapse within a few $\times 10^{8}$ yrs of a massive protogalaxy with concurrent rapid chemical enrichment [Eggen et al., 1962 (hereinafter ELS); Sandage, 1990]; and

- Merger over time of subunits that had undergone independent chemical evolution [Searle and Zinn, 1978 (hereinafter SZ)].

As most recently summarized by Armandroff (1993), Kinman's (1959) suspicion that the GCs comprise two kinematical and chemical subsystems is now well established, with the division occurring near $[\mathrm{Fe} / \mathrm{H}] \sim-0.8$ (i.e., between the slowly rotating clusters of spectral type $F$, and the more rapidly rotating ones of type $G$ ). The remainder of this discussion focusses on efforts to determine relative ages within the subsystems of the GCs as a function of $R_{g c}$.

\subsection{RELATIVE AGES FROM MAIN SEQUENCES}

The position and comportment of the age-sensitive turnoff from the hydrogen burning main sequence is sensitive to chemical composition, but the exact dependence is a matter of debate. Over and above the impact of the overall $[\mathrm{Fe} / \mathrm{H}]$ ratio, the ranges within a single cluster of lighter elements (§3.1) represent uncertainties in age estimation that are rarely dealt with explicitly. 
Age determinations using the turnoff rely typically on three methods: a) challenging absolute measurements, such as in the comparison of NGC 288 and NGC 362 (e.g., Bolte, 1989, 1993; Green and Norris, 1990); b) analysis of the luminosity differences between the $\mathrm{HB}$ and main-sequence turnoff, $\Delta \mathrm{V}(\mathrm{HB}-\mathrm{TO})$; and $\mathrm{c})$ the color difference between the turnoff and the base of the red-giant branch, $\Delta(\mathrm{B}-\mathrm{V})(\mathrm{MS}-\mathrm{RGB})$ [for a summary of the latter two, see Salaris et al., 1993). The $\Delta \mathrm{V}(\mathrm{HB}-\mathrm{TO})$ method benefits from a presumably sounder theoretical basis for calibration through models, as luminosities are more reliably predicted than the radii essential for colors. However, method c) is operationally more cleanly defined (see VandenBerg et al., 1990; Sarajedini and Demarque, 1990) than method b), where the distribution of stars along the HB introduces substantial difficulties.

The net application of the above methods in the past five or so years has led, for the first time, to unambiguous evidence for a detectable age range among the GCs, with Arp 2, Pal 12, Rup 106, and Ter 7 appearing to have ages 3-5 Gyrs younger than the bulk of the system (Bolte, 1993; Stetson, 1993 and references therein). None of those four clusters lies in the brighter half of the GC luminosity function, and they all lie at $\mathrm{R}_{\mathrm{gc}}>16 \mathrm{kpc}$ (a typical GC has $\mathrm{M}_{\mathrm{V}} \sim-7.3$ and half of them have $\mathrm{R}_{\mathrm{gc}}<8 \mathrm{kpc}$ ). Several of these clusters lie near the newly discovered Sagittarius dSph (Ibatta et al., 1994).

Do a few GCs younger by some $20 \%$ than the bulk spell the end of the ELS rapid collapse concept? Perhaps not yet. The data still allow the possibility of an age distribution with a narrow peak and a long, sparse tail to younger ages, i.e., ELS with a minor portion of the material dawdling at large distances. Or, perhaps, the younger clusters were captured during the tidal disruption of a dwarf galaxy, as advocated by Lin and Richer (1992). That all five of the best observed metal-poor GCs have the same age to within $0.5 \mathrm{Gyr}$ is statistically remarkable if the GC system has a normally distributed age distribution with $\sigma \sim 3$ Gyr (Bolte, 1993; Stetson, 1993).

To this point unambiguous evidence favoring one Galactic formation scenario over another hasn't emerged, although Suntzeff, et al.'s (1991) and Armandroff, et al.'s (1992) findings of a metallicity gradient interior to $\mathrm{R}_{\mathrm{gc}} \sim 8 \mathrm{kpc}$ suggests that an ELS-like rapid collapse might have dominated in the inner halo, while a SZ accretion or merger picture obtained in the outer regions. Earlier, I intentionally belabored possible evolutionary modfications of GC properties to sensitize us towards uncertainties in global inferences about Galactic formation. But a dominant theme in current GC research has been neglected in the foregoing: for better or for worse, we can no longer avoid entering the heart of the forbidding "second-parameter jungle". 


\subsection{INFERENCES FROM THE SECOND PARAMETER}

Van den Bergh (1965) and Sandage and Wildey (1967) called attention to the role of some factor other than a monotonic dependence on $[\mathrm{Fe} / \mathrm{H}]$ governing the morphological differences among GC HBs. Present theory indicates that distributions of stars on HBs can be dramatically affected by changes smaller than observers can measure for parameters such as $\mathrm{Y}$, [C$\mathrm{NO} / \mathrm{Fe}],[\alpha / \mathrm{Fe}], \mathrm{M}_{\text {core }} / \mathrm{M}_{\text {tot }}$, core rotation..., while observations (Fusi Pecci et al., 1993) hint at links between dynamical history and HB morphologies, as well. Rood's (1973) suggestion of age as a second parameter receives increasing attention.

From comparisons of synthetic HB models with a modified Mironov parameter characterizing the observed distribution of $\mathrm{HB}$ stars, Lee et al.(1994) have built a compelling case for the clusters with $\mathrm{R}_{\mathrm{gc}} \leqslant 8 \mathrm{kpc}$ being, on average, older by some $2 \mathrm{Gyr}$ than those with $8 \leq \mathrm{R}_{\mathrm{gc}}<40 \mathrm{kpc}$, which in turn are found to be some 2 Gyr older than outer halo clusters beyond $40 \mathrm{kpc}$. In the latter group, the second parameter dominates: the bulk of the clusters have red HBs, although their overall metallicities are down considerably more than a factor ten from solar. Lee (1992) argues further for a clear "inside out" formation process, in which the bulge RR Lyrae stars formed $\sim 1 \mathrm{Gyr}$ before the inner $\left(\mathrm{R}_{\mathrm{gc}}<8 \mathrm{kpc}\right)$ halo, although his conclusion is controversial (e.g., Holzman et al., 1993; McWilliam and Rich, 1994).

A provocative, alternate synthetic HB analysis by Catelan and De Freitas Pacheco (1993) stresses anew the dependence of the second parameter on differences (far below present thresholds for direct measurement) of such things as $\mathrm{Y}_{\mathrm{MS}},[\alpha / \mathrm{Fe}]$, and differential mass loss along the red-giant branch. They argue that the nature and magnitude of the problem depends on the absolute values of such parameters, as well, with present stellar models implying absolute ages < 10 Gyr for NGC 288 and NGC 362 if their relative ages differ by $\sim 3$ Gyr. Since derivations of absolute ages find most of the metal-deficient GCs to be 15-17 Gyr old, their remarks are sobering.

Ignoring for now caveats on interpretation of the second parameter, let's return to the theme of distinct subsystems within the GC system as a whole. Rodgers and Paltoglou (1984) called attention to the possibility that some GCs having $-1.7 \approx[\mathrm{Fe} / \mathrm{H}] \approx-1.3$ rotate about the Galactic center in a retrograde sense, which suggests they might have been accreted during the absorption of a victim galaxy. Many of the clusters with large retrograde motions have unusually red HBs. Van den Bergh (1993) and Zinn (1993) independently extended this concept and strengthened the link between the second parameter and cluster kinematics. They find that halo GCs (as distinct from the nuclear or disk subsystem) interior to $\sim 8 \mathrm{kpc}$ are on more circular orbits and exhibit a smaller inferred (largely from synthetic HB 
modelling) age range, while many of the halo GCs beyond $\mathrm{R}_{\mathrm{gc}} \sim 8 \mathrm{kpc}$ are on plunging, retrograde orbits and exhibit a greater inferred age range. An important difference in these views is the mass of the proposed ancestral galaxies, with $\mathrm{SZ}$ and Zinn advocating numerous objects with $\sim 10^{7} \mathrm{M}_{\odot}$, while van den Bergh favors one or more objects a thousand times more massive.

We have seen evidence: a) for some GCs with ages $\sim 20 \%$ less than the bulk of the system [arising principally from application of the $\Delta \mathrm{V}(\mathrm{HB}-\mathrm{TO})$ and $\Delta(\mathrm{B}-\mathrm{V})$ (MS-RGB) methods]; b) indirectly (from synthetic HB modelling), for an $R_{\mathrm{gc}}$ dependence in which the oldest clusters are found nearer to the Galactic center; and c) for three kinematic subsystems (disk/nuclear bulge, and inner- and outer-halo). What other clues regarding age as a function of $R_{\mathrm{gc}}$ might guide our understanding of how the Galaxy formed?

\subsection{AGE DETERMINATIONS UNDERWAY}

In $\S 2$ GCs and dSphs were briefly contrasted. Programs in progress allow accurate comparisons to be made for the first time between such objects at $R_{\mathrm{gc}} \sim 100 \mathrm{kpc}$. Fig. 1 is a preliminary CMD for the Carina dSph obtained by Smecker-Hane et al.(1994a) using the CTIO 4-m telescope and ALLFRAME (Stetson, 1994). Multiple turnoffs feed stars onto a remarkably narrow red-giant branch. Ages inferred using Revised Yale Isochrones (Green et al., 1987) are, roughly, 12-15 Gyr, 5-8 Gyr, 4 Gyr and, possibly, $\sim 2$ Gyr (the latter may equally represent a strong blue-straggler sequence). These results reveal the progenitors of features seen in CMDs for more luminous Carina stars (Mighell, 1990; Smecker-Hane et al., 1994b), e.g., the clump of luminous red HB stars present simultaneously with a traditional GC extended HB. Such results further demonstrate a complex starformation history for this modest object presently located at $\mathrm{R}_{\mathrm{gc}} \sim 105 \mathrm{kpc}$ in the outer Galactic halo.

At similar $\mathrm{R}_{\mathrm{gc}}$, CFHT observations for the GCs Pal 4 and NGC 2419 (Christian and Heasley, 1986, 1988) showed them to be each of a single age and old, but were unable to constrain particularly quantitatively our understanding of the Galactic age profile. For the first time, HST observations promise to do so (Fig. 2). From preliminary analysis of a subset of data from WFPC2, Pal 4 and NGC 2419 appear to have $\Delta$ (HB-TO) values consistent with their being coeval, as well as similar in age to clusters in the inner halo.

What of clusters in the inner halo? During a June, 1994 CFHT observing run, Stetson and VandenBerg (private communication) performed a fully differential comparison (similar to Bolte, 1989) of the classic northern hemisphere second-parameter pair, M3 and M13. To within observational 


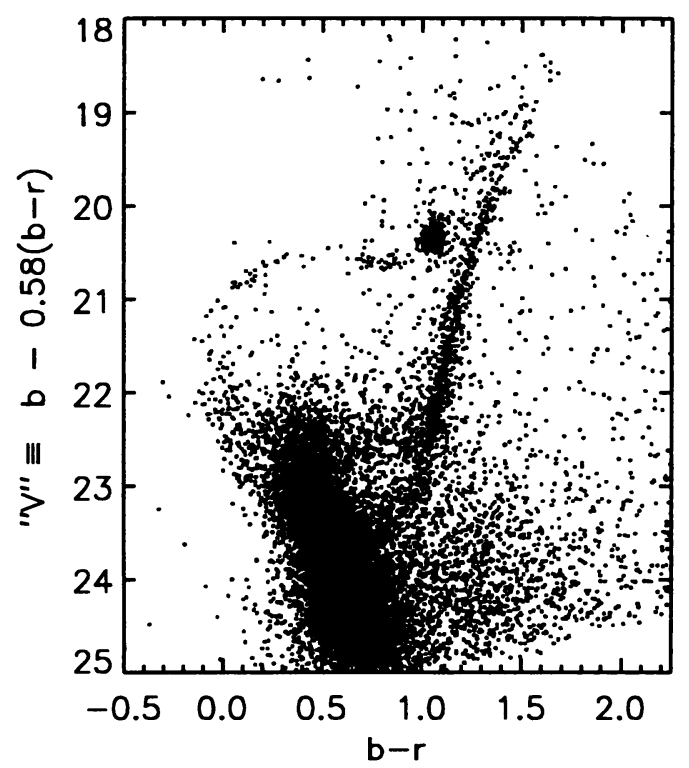

Figure 1. A preliminary CMD for $\sim 18,000$ stars the Carina dSph (Smecker-Hane et al., 1994a).
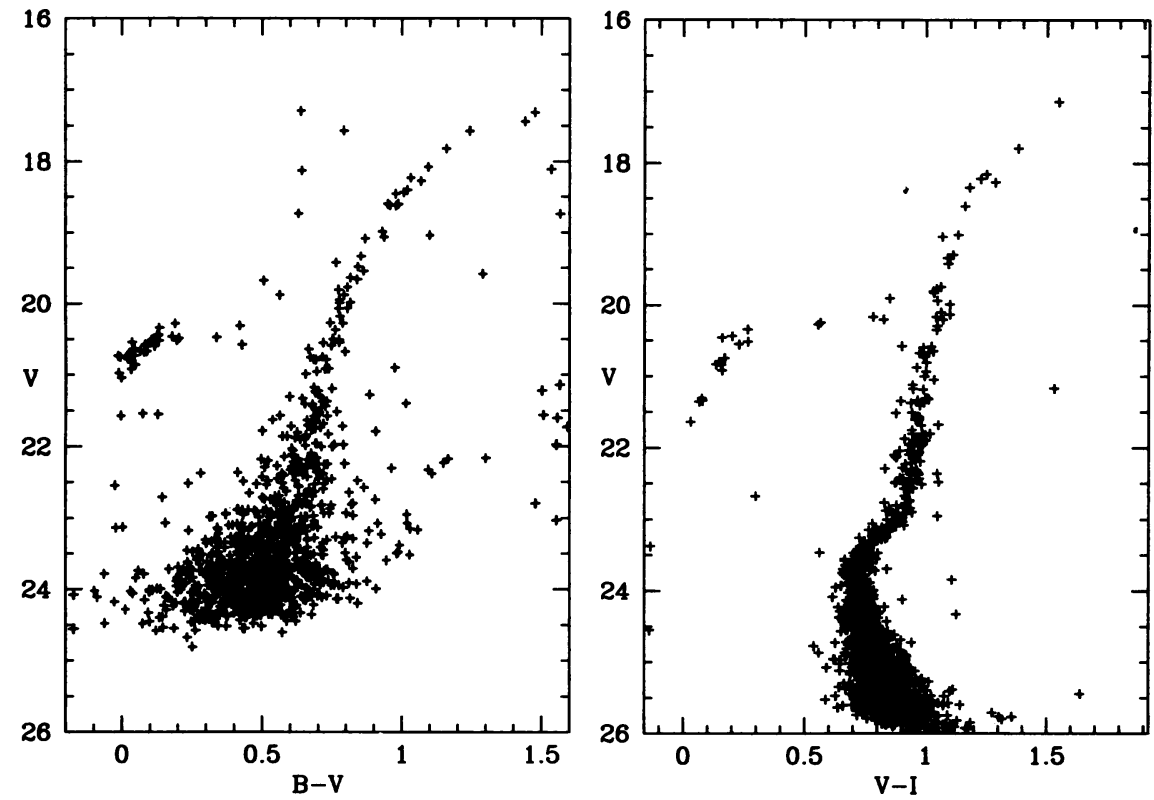

Figure 2. (Left) CFHT observations of NGC 2419 (Christian and Heasley, 1988); (right) a preliminary CMD from HST WFPC2 data (Stetson et al., 1994). The clarity of the HST CMD promises to facilitate age determination for such distant, crowded fields. 
errors, these clusters have identical global chemical properties (e.g., Kraft et al., 1993). If age is the second parameter, then M13 should be approximately 3 Gyr older than M3. Preliminary interpretation of the CFHT B,I photometry suggests that these two clusters have the same age to within $0.8 \mathrm{Gyr}$. Kraft, et al.point out that M13 has six stars on the first ascent of the giant branch that are very deficient in oxygen. They speculate that the brightest M13 giants might have envelopes about $15 \%$ less massive than comparable M3 stars. Perhaps angular momentum regulates deep mixing at the advanced stages of evolution, such that it, rather than age differences, drives the observed differences in HB morphology.

The advent of CCDs led to renewed attempts to observe the GCs viewed against the rich star fields of the nuclear bulge, but even the most heroic efforts from the ground (e.g., Sarajedini and Norris, 1994) provide little hope of measuring the main-sequence turnoff region with sufficient accuracy to determine precise ages. Again, however, HST promises to provide the long-sought answer as to whether there is significant age spread amongst the nuclear bulge clusters and to whether or not they are significantly older than their counterparts in the outer halo (Fullton and Carney, this Symposium).

We asked: How did the system form and what does that tell us about how large spiral galaxies form? The answer clearly remains to be determined, but, with HST and the best ground-based telescopes, the answer is, for the first time in my career, tantalizingly close. At this stage in our quest we have evidence for:

- Subsystems (kinematic, chemical) comprising the GCs of G and F spectral type, with the latter apparently subdivided into retrograde and prograde (younger, older?) families.

- The second parameter being exaggerated in the outer halo GCs and $\mathrm{dSphs}$; if this effect arises predominantly from age, then, on average, those systems should be younger and exhibit a greater age spread than GCs at smaller $\mathrm{R}_{\mathrm{gc}}$.

- Five GCs with $[\mathrm{Fe} / \mathrm{H}] \lesssim-2$ have age differences $<0.5$ Gyr, while a few intermediate-metallicity GCs appear to be younger than the bulk of the halo GCs by $\sim 20 \%$.

- Multiple stellar generations spanning some 8 Gyrs in the Carina dSph contrasts with single-age GCs at the same $R_{g c}$.

- An ELS-like, rapid collapse phenomenon at $\mathrm{R}_{\mathrm{gc}}<8 \mathrm{kpc}$, and a SZ-like merger beyond, but in all likelihood these remain overly simplistic views of how the Galaxy really formed.

The refurbished HST is demonstrably capable of providing superb CMDs for use in determing accurate relative ages of nuclear and halo GCs; likely some surprises are still in store as age is evaluated as the dominant second parameter: stay tuned! 


\section{Population II and High Redshift Astrophysics}

There are many exciting things to do in astronomy and I, for one, cannot make the time to work in all the areas that intrigue me. As I ponder mysteries of GCs, I sometimes get the feeling (perhaps some of you do, too?) that I'm not doing real astronomy (e.g., the quest for high redshift QSOs, primeval galaxies, etc.). I take some heart, however, when recalling numerous studies (e.g., Pettini, et al., 1994) suggesting that damped Lyman- $\alpha$ systems in quasars with $1.5<\mathrm{z}<3.5$ have metallicities down from solar by only 1.5 dex or so. Tonight, however, armed only with a pair of binoculars and a chart from any good sky atlas, you can view a majestic object whose chemistry suggests it formed at $\mathrm{z}=3,4,5 \ldots: \mathrm{M} 15,[\mathrm{Fe} / \mathrm{H}]=-2.2$, and only a few kpc distant! It, and its counterparts throughout the halo, still merit attention from observers and theoreticians if we are to understand Baade's Population II and its role in how the Galaxy, and perhaps other large spirals, formed.

Acknowledgement: I thank the IAU for travel support, numerous colleagues for their suggestions, and R. McClure, T. Smecker-Hane, P. Stetson and S. van den Bergh for commenting upon the draft manuscript.

\section{References}

Aguilar, L.A., Hut, P. and Ostriker, J.P., 1988, Ap. J. 335, 720

Armandroff, T., 1993, ASP Conf. Series 48, 48

Armandroff, T., Da Costa, G.S. and Zinn, R., 1992, A. J. 104, 164

Baade, W., 1944, Ap. J. 160, 137

Bolte, M., 1989, A. J. 97, 1688

Bolte, M., 1993, ASP Conf. Series 48, 60

Briley, M.B., Bell, R.A., Hoban, S. and Dickens, R.J., 1990, Ap. J. 359, 307

Briley, M.B., Bell, R.A., Smith, G.H. and Hesser, J.E., 1994a, C. J. P., December

Briley, M.B., Hesser, J.E., Bell, R.A., Bolte, M. and Smith, G.H., 1994b, A. J. December

Catelan, M. and De Freitas Pacheco, J.A., 1993, A. J. 106, 1858

Chernoff, D.F. and Weinberg, M., 1990, Ap. J. 351, 121

Christian, C.A. and Heasley, J.N., 1986, Ap. J. 303, 216

Christian, C.A. and Heasley, J.N., 1988, A. J. 95, 1422

Denisenkov, P.A. and Denisenkova, S.N., 1990, Sov. Astron. L. 16, 275

Djorgovski, S.G. and Meylan, G., 1994, A. J. 108, 1292

Dubath, P., Meylan, G. and Mayor, M., 1992, Ap. J. 400, 510

Durrell, P. and Harris, W.E., 1993, A. J. 105, 142

Eggen, O.J., Lynden-Bell, D. and Sandage, A., 1962, Ap. J. 136, 748 (ELS)

Fall, S.M., 1980, Globular Clusters, eds. D.A. Hanes and B. Madore, Cambridge U. Press, p.309

Fusi Pecci, F. et al., 1993, A. J. 105, 1145

Green, E.M. and Norris, J.E., 1990, Ap. J. 353, L17

Green, E.M., Demarque, P. and King, C.R., 1987, The Revised Yale Isochrones, Yale University Observatory, New Haven

Harris, W.E., 1987, ESO Conf. Proc. 27, eds. M. Azzopardi and F. Matteuci, Garching, ESO, p.683

Harris, W.E., 1991, Ann. Rev. Astron. Astroph. 29, 543 
Hesser, J.E., Shawl, S.J.and Meyer, J.E., 1986, P. A. S. P. 98, 403

Holzman, J.A. et al., 1993, A. J. 106, 1826

Ibata, R.A., Gilmore,G. and Irwin, M.J., 1994, Nature 370, 194

Kinman, T.D., 1959, M. N. R. A. S. 119, 538

Kraft, R.P., 1994, P. A. S. P. 106, 553

Kraft, R.P., Sneden, C., Langer, G.E. and Shetrone, M.D., 1993, A. J. 106, 1490

Kumai, Y., Basu, B. and Fujimoto, M., 1993, Ap. J. 404, 144

Laird, J.B., Carney, B.W. and Latham, D.W., 1993, ASP Conf. Series 48, 95

Langer, G.E., Hoffman, R.D. and Sneden, C., 1993, P. A. S. P. 105, 301

Lee, Y-W., 1992, A. J. 104, 1780

Lee, Y-W., Demarque, P. and Zinn, R., 1994, Ap. J. 423, 248

Lin, D.N.C. and Richer, H.B., 1992, Ap. J. 388, L57

McWilliam, A. and Rich, M., 1994, Ap. J. Suppl. 91, 749

Meylan, G. and Pryor, C., 1993, ASP Conf. Series 50, 31

Mighell, K.J., 1990, A. \& A. 82, 1

Norris, J.E. and Freeman, K.C., 1982, Ap. J. 277, 615

Pettini, M., Smith, L.J., Hunstead, R.W. and King, D.L., 1994, Ap. J. 426, 79

Ortolani, S., Bica, E. and Barbuy, B., 1993, A. \& A. 267, 66

Pryor, C., McClure, R.D., Fletcher, J.M. and Hesser, J.E., 1991, A. J. 102, 1026

Racine, R. and Harris, W.E., 1989, A. J. 98, 1609

Rodgers, A.W. and Paltoglou, G., 1984, Ap. J. 283, L5

Rood, R., 1973, Ap. J. 184, 815

Saha, A., 1985, Ap. J. 289, 310

Salaris, M., Chieffi, A. and Straniero, O., 1993, Ap. J. 414, 580

Sandage, A., 1990, J. R. A. S. Can. 84, 70

Sandage, A. and Wildey, R., 1967, Ap. J. 150, 469

Sarajedini, A. and Demarque, P., 1990, Ap. J. 365, 219

Sarajedini, A. and Norris, J.E., 1994, Ap. J. Suppl. 93, 161

Searle, L., 1977, The Evolution of Galaxies and Stellar Populations, eds. B.M. Tinsley and R.B. Larson, New Haven: Yale U. Obs., 219

Searle, L. and Zinn, R., 1978, Ap. J. 225, 357 (SZ)

Smecker-Hane, T.A., Stetson, P.B. and Hesser, J.E., 1994a, in preparation

Smecker-Hane, T.A., Stetson, P.B., Hesser, J.E. and Lehnert, M.D., 1994b, A. J. 108, 507

Smith, G.H., 1987, P. A. S. P. 99, 67

Smith, G.H. and Wirth, G.D., 1991, P. A. S. P. 103, 1158

Stetson, P.B., 1993, ASP Conf. Series 48, 14

Stetson, P.B., 1994, P. A. S. P. 106, 250

Stetson, P.B. et al., 1994, in preparation

Suntzeff, N.B., 1993, ASP Conf. Series 48, 167

Suntzeff, N.B., Kinman, T.D. and Kraft, R.P., 1991, Ap. J. 367, 528

VandenBerg, D.A., 1988, IAU Symp. 126, 107

VandenBerg, D.A., Bolte, M. and Stetson, P.B., 1990, A. J. 100, 445

van den Bergh, S., 1965, J. R. A. S. Can. 59, 151

van den Bergh, S., 1993, Ap. J. 411, 178

van den Bergh, S., 1994, Ap. J. 435, 203

van den Bergh, S., Morbey, C. and Pazder, J., 1991, Ap. J. 375, 594

Zinn, R., 1985, Ap. J. 293, 42

Zinn, R., 1993, ASP Conf. Series 48, 38

BELL: I think that your comments on the uncertainty in cluster ages arising from the possibility of high oxygen abundances is unduly pessimistic. The VandenBerg calculations used a very high $\mathrm{O}$ abundance which is no longer accepted. 
This abundance was never in the cards, anyway, since it would lead to great inconsistencies in the strength of the $\mathrm{CH}$ and $\mathrm{CO}$ bands in the luminous giants. The $\mathrm{C}$ abundances needed to fit the $\mathrm{CH}$ bands would give $\mathrm{CO}$ bands much stronger than those observed. If the $\mathrm{O}$ had been transformed to $\mathrm{N}$, the predicted $\mathrm{CN}$ band strengths would be too strong.

HESSER: Agreed. I was trying to make two points in my hurried summary. First, that while attention was first called to the possible importance of using O-enhanced isochrones for interpreting the CMDs of Galactic globular clusters, some now argue that $\alpha$-enhanced isochrones are more appropriate. Second, and most importantly, observers are able to measure detailed abundances only for luminous giants. It is now clear thate their abundance ratios have been significantly altered (for CNO, $\mathrm{Ca}, \mathrm{Al}$ ) as a result of stellar evolutionary processes. They do not represent the values appropriate to interpretation of CMDs in terms of ages, which is the principal focus of my discussion here. We all hope that the Keck telescope will devote many hours to HiRes studies of abundances for faint globular clusters, so we can all put this problem to rest! 